Supporting Information

\title{
A Molecular Ferroelectric with Most Equivalent Polarization Directions Induced by the Plastic Phase Transition
}

Heng-Yun Ye*, Jia-Zhen Ge, Yuan-Yuan Tang, Peng-Fei Li, Yi Zhang, Yu-Meng You*, Ren-Gen Xiong*

Supplementary Methods: Differential scanning calorimetry (DSC), SHG, dielectric, pyroelectric, $P-$ $E$ hysteresis loops measurements were described elsewhere ${ }^{\mathrm{S} 1}$. For dielectric measurements, powder-pressed pellets with about $5 \mathrm{~mm}^{2}$ in area and $0.5 \mathrm{~mm}$ in thickness were used. Silver conduction paste deposited on the plate surfaces was used as the electrodes.

For $P-E$ hysteresis loop measurements, a thin-film sample was fabricated. The detailed process is described as follows. Firstly, commercial ITO-coated glass substrate $(\Phi=15 \mathrm{~mm})$ was sequentially ultrasonic cleaned in toluene, acetone, ethanol and deionized water 20 minutes at a time. A drop of precursor solution contains $\mathbf{1}(100 \mathrm{mg} / \mathrm{ml})$ was carefully spread on a freshly cleaned ITO-coated glass (conductive ITO layer was used as the bottom electrode). With controlled substrate temperature $\left(\sim 65^{\circ} \mathrm{C}\right)$ and edge-pinned-crystallization, a uniform film consists of continuous dendritic crystal with high coverage was obtained. After that then liquid GaIn eutectic was used directionally as the top electrode, then all ferroelectric hysteresis measurements were conducted with this sandwich architecture (GaIn/Sample film/ITO) using the standard double-wave method. The film thickness was measured with a manmade gap using AFM of around $3 \mu \mathrm{m}$.

Nanoscale polarization imaging and local switching spectroscopy were carried out using a resonant-enhanced piezoresponse force microscopy (MFP-3D, Asylum Research). Conductive Pt/Ir-coated silicon probes (EFM-50, Nanoworld) were used for domain imaging and polarization switching studies. To verify the piezoresponse, we applied a $10 \mathrm{~V}$ AC driving voltage on the sample 
to measure the normal and shear responses, with the AC frequency set at the second resonant peak of cantilever-sample system (340 kHz for normal response and $720 \mathrm{kHz}$ for shear response) to enhance the sensitivity.

Supplementary Figures:

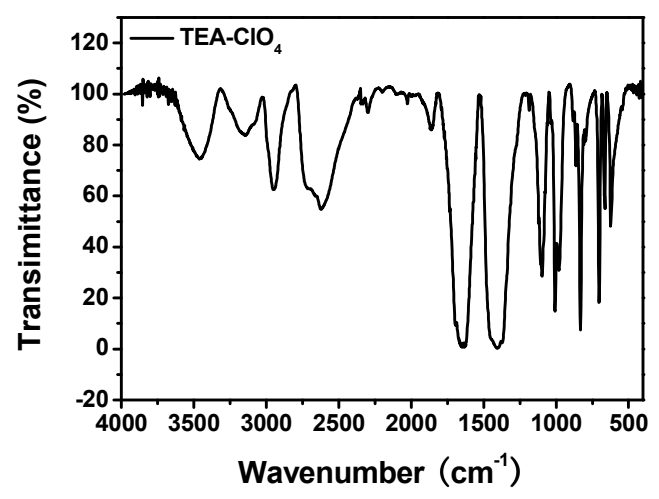

Figure S1. Infrared spectrum for $\mathbf{1}$

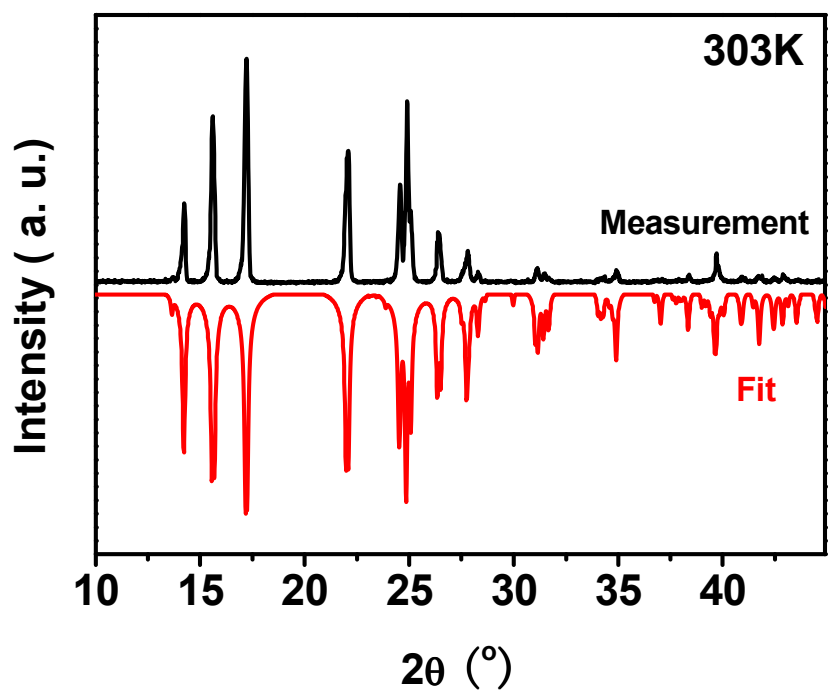

Figure S2. PXRD pattern of $\mathbf{1}$, verifying the purity of the bulk phase. 


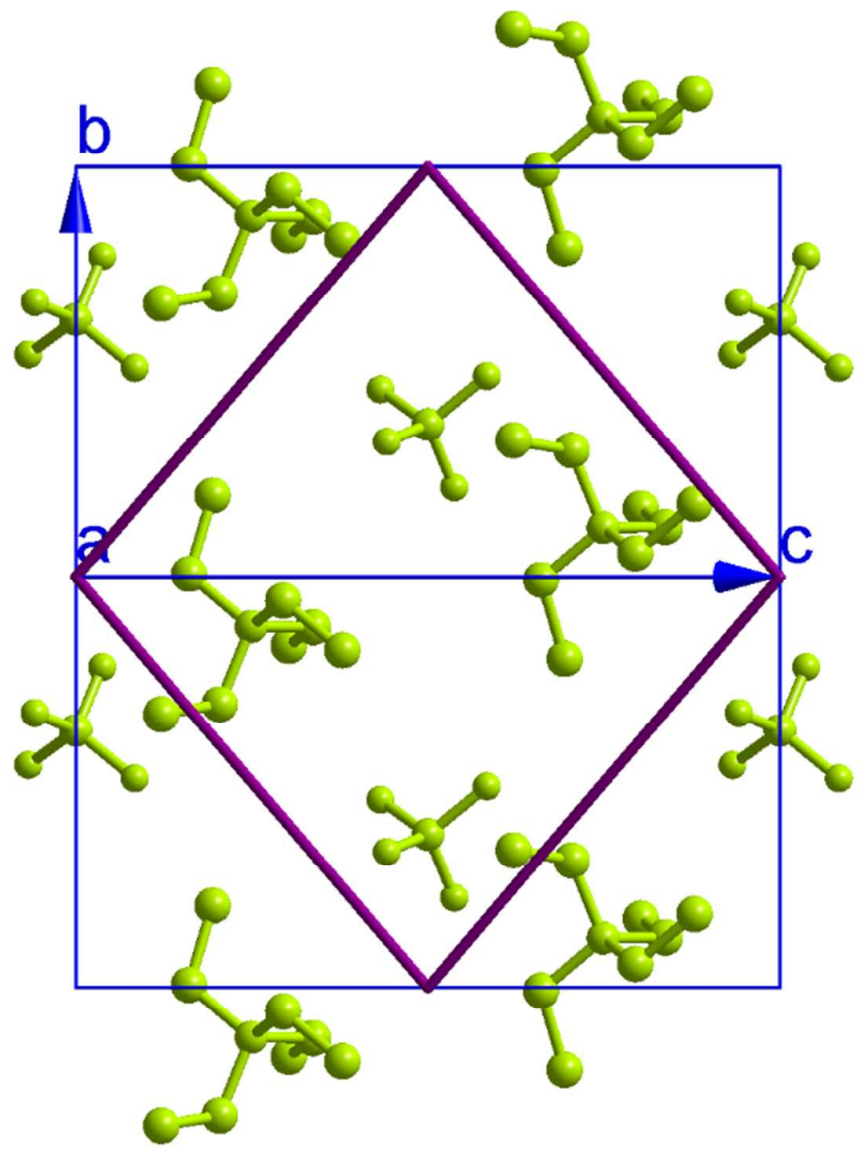

Figure S3. A layer in the $\left[\begin{array}{lll}1 & 0 & 0\end{array}\right]$-direction of $\mathbf{1}$, Showing the similarities to and differences from that of a $\left[\begin{array}{lll}1 & 0 & 0\end{array}\right]$ layer in $\mathrm{NaCl}$. 

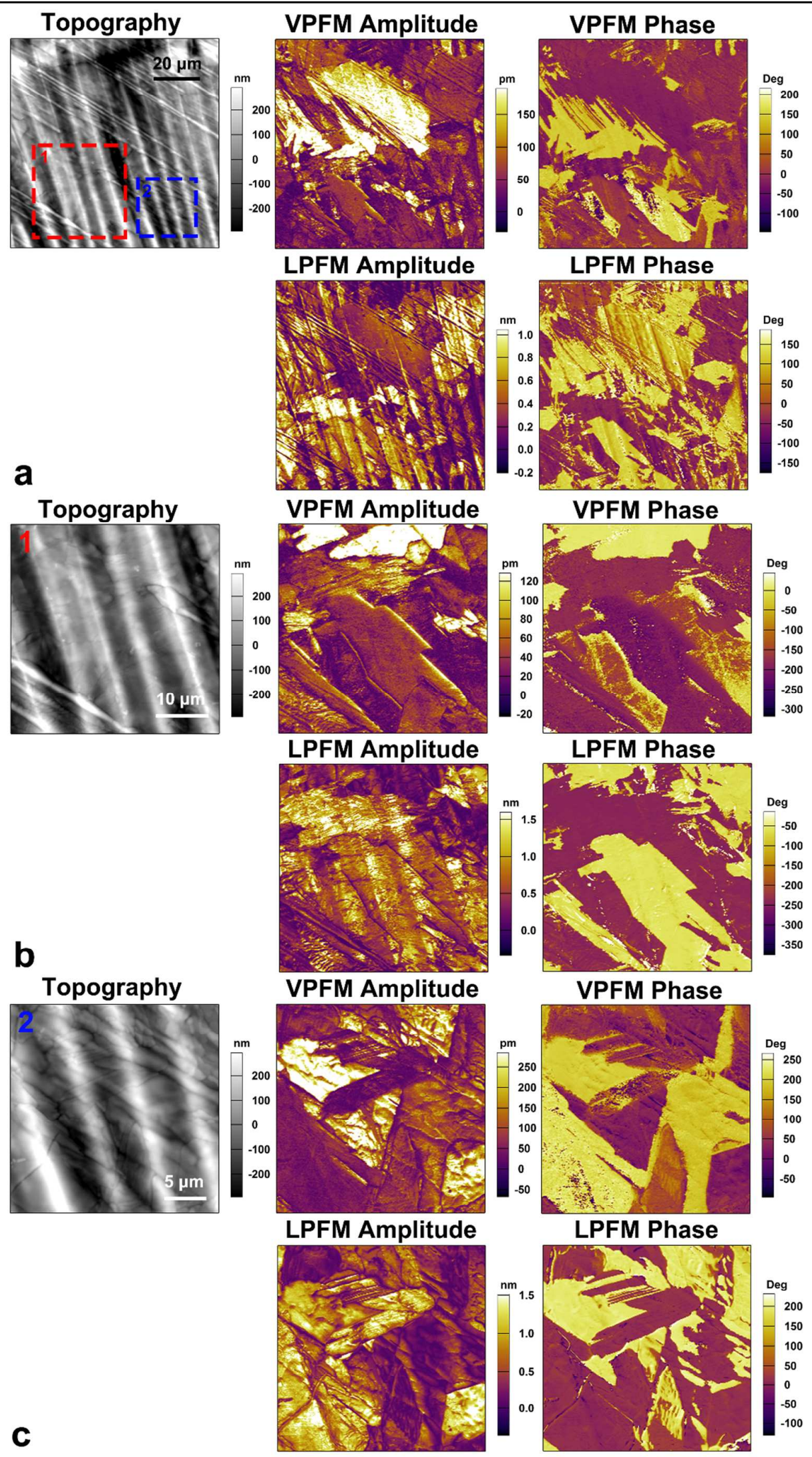

Figure S4. Vertical and Lateral PFM images and topographic images for the film surface $\mathbf{1}$ over areas of (a) $90 \mu \mathrm{m} \times 90 \mu \mathrm{m}$, (b) $40 \mu \mathrm{m} \times 40 \mu \mathrm{m}$ and (c) $25 \mu \mathrm{m} \times 25 \mu \mathrm{m}$, respectively. The regions of (b) and (c) are parts of (a). As can be seen, the domain distributions in the two modes are different. We 
first scan a region over $90 \mu \mathrm{m} \times 90 \mu \mathrm{m}$ (Figure S4a). The phase and amplitude images show various contrasts, indicating many polarization directions in the different domains. The scanned area was then zoomed out in two small areas of $40 \mu \mathrm{m} \times 40 \mu \mathrm{m}$ and $25 \mu \mathrm{m} \times 25 \mu \mathrm{m}$ respectively. The domain patterns shown in Figure S4b \& S4c are consistent with the corresponding regions in Figure S4a, further revealing various non- $180^{\circ}$ domain structures. The adjacent domains with different polarization directions form vast amounts of domain patterns, some of which are very unique. These results present direct experimental proofs for the presence of non- $180^{\circ}$ domains in $\mathbf{1}$.

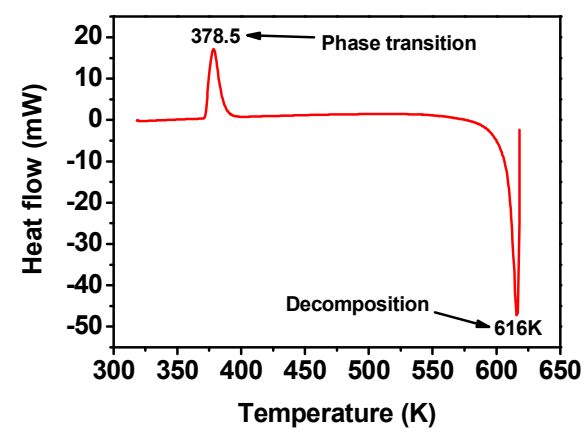

Figure S5. Thermal analysis for 1, revealing the good thermal stability up to $616 \mathrm{~K}$.

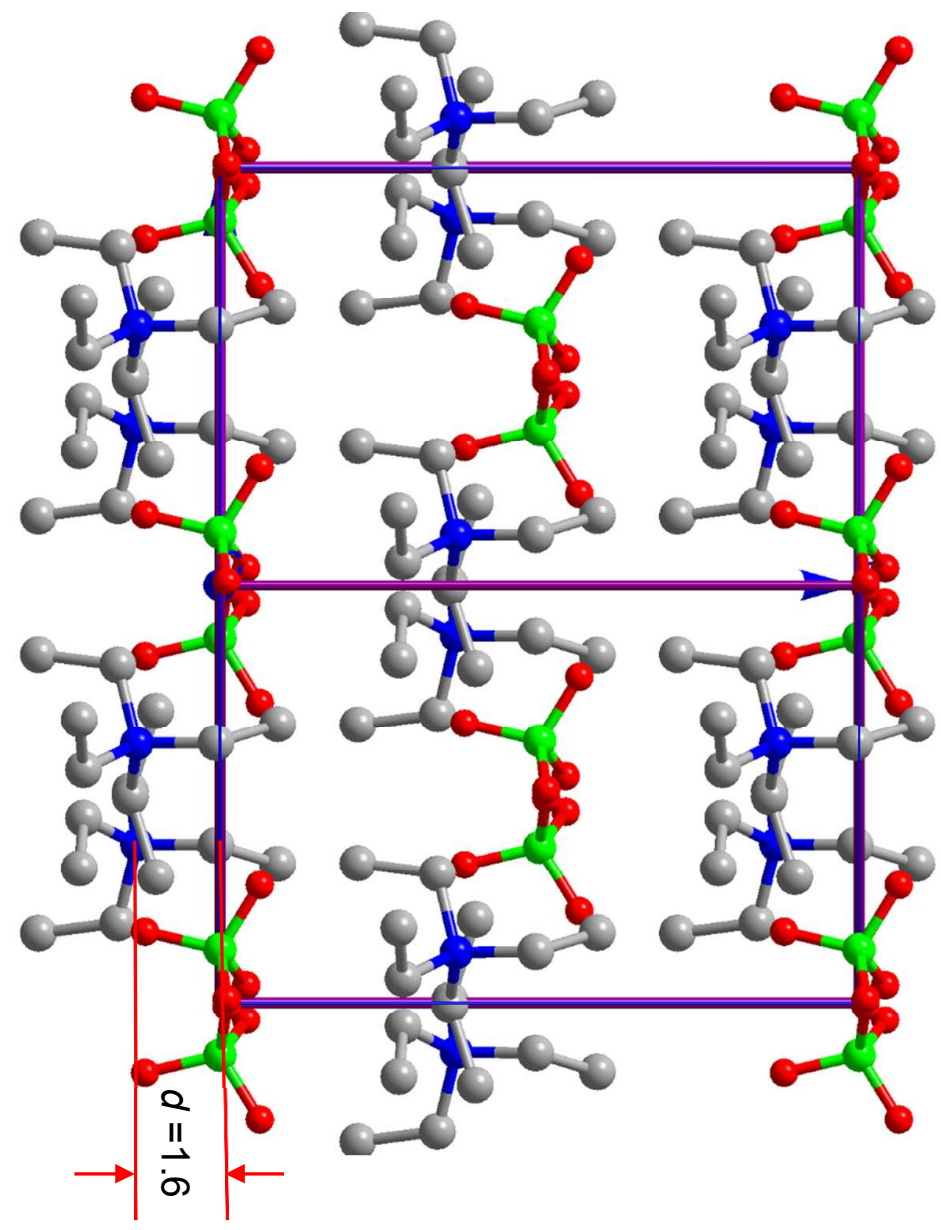

Figure S6. Projection of $\mathbf{1}$ along the $c$-direction, showing the relative displacement of the cations and anions in the same layers along the $a$-direction. 


\section{Supplementary discussion}

Point charge model analysis: According to the crystal structure data at $293 \mathrm{~K}$, we select a unit cell and make an assumption that the positive charge of $\left(\mathrm{C}_{8} \mathrm{H}_{20} \mathrm{~N}\right)^{+}$and the negative charge of $\left(\mathrm{ClO}_{4}\right)^{-}$is on the $\mathrm{N}$ atom and $\mathrm{Cl}$ atom, respectively.

\begin{tabular}{|c|c|r|r|}
\hline \multirow{4}{*}{ Atoms } & \multicolumn{2}{|c|}{ Coordinate } & Center coordinate \\
\hline \multirow{4}{*}{$\mathrm{N}$} & $\mathrm{N} 1^{1}(0.3735,0.8775,0.2499)$ & $\mathrm{N} 1^{2}(0.3735,-0.1225,0.2499)$ & \\
\cline { 2 - 4 } & $\mathrm{N} 2^{1}(0.3735,1.1225,0.7499)$ & $\mathrm{N} 2^{2}(0.3735,0.1225,0.7499)$ & \multirow{2}{*}{$(0.3735,0.5,0.4999)$} \\
\cline { 2 - 4 } & $\mathrm{N} 3^{1}(0.8735,0.3775,0.2499)$ & $\mathrm{N} 3^{2}(-0.1265,0.3775,0.2499)$ & \\
\cline { 2 - 4 } & $\mathrm{N} 4^{1}(0.8735,0.6225,0.7499)$ & $\mathrm{N} 4^{2}(-0.1265,0.6225,0.7499)$ & \\
\hline \multirow{4}{*}{$\mathrm{Cl}$} & $\mathrm{Cl} 1^{1}(1,0.13012,0)$ & $\mathrm{Cl} 1^{2}(1,0.13012,1)$ & \\
\cline { 2 - 4 } & $\mathrm{Cl} 1^{3}(0,0.13012,0)$ & $\mathrm{Cl} 1^{4}(0,0.13012,1)$ & \multirow{2}{*}{$(0.5,0.5,0.5)$} \\
\cline { 2 - 4 } & $\mathrm{Cl} 2^{1}(1,0.86988,0.5)$ & $\mathrm{Cl} 2^{2}(0,0.86988,0.5)$ & \\
\cline { 2 - 4 } & $\mathrm{Cl} 3^{1}(0.5,0.63012,0)$ & $\mathrm{Cl} 3^{2}(0.5,0.63012,1)$ & \\
\cline { 2 - 4 } & $\mathrm{Cl} 1(0.5,0.36988,0.5)$ & & \\
\hline
\end{tabular}

$$
\begin{aligned}
& P=\lim \frac{1}{V} \Sigma q_{\mathrm{i}} r_{\mathrm{i}} \\
& =\left(q_{\mathrm{N}} r_{\mathrm{N}}+q_{\mathrm{Cl}} r_{\mathrm{Cl}}\right) / \mathrm{V} \\
& =[(\mathrm{e} \times 0.3735)+(-\mathrm{e} \times 0.5)] \times 4 \times \mathrm{c} / \mathrm{V} \\
& =\left[0.1265 \times 4 \times 1.6 \times 10^{-19} \times 12.61 \times 10^{-10} \mathrm{C} \mathrm{m}\right] /\left(1217.02 \times 10^{-30} \mathrm{~m}^{3}\right) \\
& =8.39 \times 10^{-2} \mathrm{C} \mathrm{m}^{-2}=8.39 \mu \mathrm{C} \mathrm{\textrm {cm } ^ { - 2 }}
\end{aligned}
$$


Supplementary References

(S1) (a) Ye, H.-Y.; Li, S. H.; Zhang, Y.; Zhou, L.; Deng, F.; Xiong, R.-G. J. Am. Chem. Soc. 2014, 136, 10033; (b) Zhang, Y.; Liu, Y. M.; Ye, H.-Y.; Fu, D. W.; Gao, W. X.; Ma, H.; Liu, Z. G.; Liu, Y. Y.; Zhang, W.; Li, J. Y.; Yuan, G. L.; Xiong, R.-G. Angew. Chem. Int. Ed. 2014, 53, 5064; (c) Liao, W. Q.; Zhang, Y.; Hu, C. L.; Mao, J. G.; Ye, H.-Y.; Li, P. F.; Huang, S. D.; Xiong, R.-G. Nat. Commun. 2015, 6,7338 .

(S2) (a) Tian, Y.; Stroppa, A.; Chai, Y. S.; Barone, P.; Perez-Mato, M.; Picozzi, S.; Sun, Y. Phys. Status Solidi-R 2015, 9, 62; (b) Tian, Y.; Stroppa, A.; Chai, Y. S.; Yan, L. Q.; Wang, S. G.; Barone, P.; Picozzi, S.; Sun, Y. Sci. Rep. 2014, 4, 6062; (c) Tian, Y.; Wang, W.; Chai, Y.; Cong, J.; Shen, S.; Yan, L.; Wang, S.; Han, X.; Sun, Y. Phys. Rev. Lett. 2014, 112, 017202; (d) Tian, Y.; Cong, J.; Shen, S.; Chai, Y.; Yan, L.; Wang, S.; Sun, Y. phys. status solidi RRL 2014, 8, 91; (e) Wang, W.; Yan, L. Q.; Cong, J. Z.; Zhao, Y. L.; Wang, F.; Shen, S. P.; Zou, T.; Zhang, D.; Wang, S. G.; Han, X. F.; Sun, Y. Sci. Rep. 2013, 3, 2024; (f) Stroppa, A.; Di Sante, D.; Horiuchi, S.; Tokura, Y.; Vanderbilt, D.; Picozzi, S. Phys. Rev. B 2011, 84, 014101; (g) Maczka, M.; Gagor, A.; Macalik, B.; Pikul, A.; Ptak, M.; Hanuza, J. Inorg. Chem. 2014, 53, 457; (h) Gomez-Aguirre, L. C.; Pato-Doldan, B.; Stroppa, A.; Yanez-Vilar, S.; Bayarjargal, L.; Winkler, B.; Castro-Garcia, S.; Mira, J.; Sanchez-Andujar, M.; Senaris-Rodriguez, M. A. Inorg. Chem. 2015, 54, 2109; (i) Maczka, M.; Pietraszko, A.; Macalik, B.; Hermanowicz, K. Inorg. Chem. 2014, 53, 787; (j) Stroppa, A.; Quarti, C.; De Angelis, F.; Picozzi, S. J. Phys. Chem. Lett. 2015, 6, 2223; (k) Ptak, M.; Maczka, M.; Gagor, A.; Sieradzki, A.; Stroppa, A.; Di Sante, D.; Perez-Mato, J. M.; Macalik, L. Dalton Trans. 2016, 45, 2574; (1) Maczka, M.; Gagor, A.; Costa, N. L. M.; Paraguassu, W.; Sieradzki, A.; Pikul, A. J. Mater. Chem. C 2016, 4, 3185; (m) Simenas, M.; Balciunas, S.; Ma Combining Cedilla Czka, M.; Banys, J.; Tornau, E. E. Phys. Chem. Chem. Phys. 2016, 18, 18528; (n) Stroppa, A.; Jain, P.; Barone, P.; Marsman, M.; Perez-Mato, J. M.; Cheetham, A. K.; Kroto, H. W.; Picozzi, S. Angew. Chem. Int. Ed. 2011, 50, 5847; (o) Gomez-Aguirre, L. C.; Pato-Doldan, B.; Stroppa, A.; Yang, L.-M.; Frauenheim, T.; Mira, J.; Yanez-Vilar, S.; Artiaga, R.; CastroGarcia, S.; Sanchez-Andujar, M.; Senaris Rodriguez, M. A. Chemistry 2016, 22, 7863; (p) Tayi, A. S.; Shveyd, A. K.; Sue, A. C. H.; Szarko, J. M.; Rolczynski, B. S.; Cao, D.; Kennedy, T. J.; Sarjeant, A. A.; Stern, C. L.; Paxton, W. F.; Wu, W.; Dey, S. K.; Fahrenbach, A. C.; Guest, J. R.; Mohseni, H.; Chen, L. X.; Wang, K. L.; Stoddart, J. F.; Stupp, S. I. Nature 2012, 488, 485; (q) Zhang, Y.; Liao, W. Q.; Fu, D. W.; Ye, H.-Y.; Liu, C. M.; Chen, Z. N.; Xiong, R.-G. Adv. Mater. 2015, 27, 3942; (r) Zhang, Y.; Liu, Y. M.; Ye, H.-Y.; Fu, D. W.; Gao, W. X.; Ma, H.; Liu, Z. G.; Liu, Y. Y.; Zhang, W.; Li, J. Y.; Yuan, G. L.; Xiong, R.-G. Angew. Chem. Int. Ed. 2014, 53, 5064; (s) Zhang, Y.; Ye, H.-Y.; Cai, H. L.; Fu, D. W.; Ye, Q.; Zhang, W.; Zhou, Q. H.; Wang, J. L.; Yuan, G. L.; Xiong, R.-G. Adv. Mater. 2014, 26, 4515; (t) Zhang, Y.; Ye, H.-Y.; Fu, D. W.; Xiong, R.-G. Angew. Chem. Int. Ed. 2014, 53, 2114. 